\title{
Determining the predominant tautomeric structure of iodine-based group-transfer reagents by ${ }^{17}$ O NMR spectroscopy
}

\author{
Nico Santschi ${ }^{\star}$, Cody Ross Pitts, Benson J. Jelier and René Verel
}

\author{
Full Research Paper \\ Address: \\ Eidgenössische Technische Hochschule (ETH) Zürich, Department of \\ Chemistry and Applied Biosciences, Vladimir-Prelog-Weg 1/2, 8093 \\ Zürich, Switzerland \\ Email: \\ Nico Santschi* - snico@inorg.chem.ethz.ch \\ ${ }^{*}$ Corresponding author \\ Keywords: \\ electrophilic; hypervalent iodine; ${ }^{17} \mathrm{O}$ NMR spectroscopy; \\ trifluoromethylation; trifluoromethylthiolation
}

Beilstein J. Org. Chem. 2018, 14, 2289-2294.

doi:10.3762/bjoc. 14.203

Received: 14 February 2018

Accepted: 23 July 2018

Published: 30 August 2018

This article is part of the Thematic Series "Hypervalent iodine chemistry in organic synthesis".

Guest Editor: T. Wirth

(C) 2018 Santschi et al.; licensee Beilstein-Institut.

License and terms: see end of document.

\begin{abstract}
Cyclic benziodoxole systems have become a premier scaffold for the design of electrophilic transfer reagents. A particularly intriguing aspect is the fundamental $\mathrm{I}^{\mathrm{I}}-\mathrm{I}^{\mathrm{III}}$ tautomerism about the hypervalent bond, which has led in certain cases to a surprising re-evaluation of the classic hypervalent structure. Thus, through a combination of ${ }^{17} \mathrm{O}$ NMR spectroscopy at natural abundance with DFT calculations, we establish a convenient method to provide solution-phase structural insights for this class of ubiquitous reagents. In particular, we confirm that Shen's revised, electrophilic $\mathrm{SCF}_{3}$-transfer reagent also adopts an "acyclic" thioperoxide tautomeric form in solution. After calibration, the approach described herein likely provides a more general and direct method to distinguish between cyclic and acyclic structural features based on a single experimental ${ }^{17} \mathrm{O}$ NMR spectrum and a computationally-derived isotropic shift value. Furthermore, we apply this structural elucidation technique to predict the constitution of an electrophilic iodine-based cyano-transfer reagent as an $\mathrm{NC}-\mathrm{I}-\mathrm{O}$ motif and study the acid-mediated activation of Togni's trifluoromethylation reagent.
\end{abstract}

\section{Introduction}

The remarkable stability and reactivity of Togni's hypervalent iodine-based trifluoromethylation reagents (e.g., 4a) [1] have inspired the development of analogous compounds, including a well-known $\mathrm{SCF}_{3}$-transfer reagent 5 in 2013 by Shen and co-workers [2,3]. In the presence of $\mathrm{AgSCF}_{3}$, chloroiodane 2a afforded an isolable and powerful electrophilic $\mathrm{SCF}_{3}$ source, which was used, for example, in $\alpha$-ketone functionalizations among other reactions $[2,3]$. While at the time the proposed cyclic hypervalent iodine structure 5a appeared reasonable in analogy to other well-established transfer reagents, it was 
unequivocally demonstrated to exist as the acyclic thioperoxide tautomer 5b by Buchwald and co-workers in 2014 [4]. The structural reassignment was prompted by a series of remarkable, detailed inspections of ${ }^{1} \mathrm{H}$ NMR spectra of precursors and congeners. A final structural corroboration came about by successfully encapsulating $\mathbf{5 b}$, an oil under ambient conditions, in a metal-organic framework (5b@MOF). This non-trivial protocol rendered it amenable to X-ray diffraction studies confirming the aforementioned structural reassignment. From a theoretical standpoint, acyclic isomer $\mathbf{5 b}$ is predicted to be thermodynamically favored over the cyclic form $\mathbf{5 a}$ by more than $10 \mathrm{kcal} / \mathrm{mol}$ by DFT calculations [5]. However, this type of computational analysis is in general still not decisive. For example, while Togni reagent 4a is thermodynamically less favorable than its acyclic isomer $\mathbf{4 b}$ by over $50 \mathrm{kcal} / \mathrm{mol}$, a high kinetic barrier suppresses the $[\mathbf{a} \rightarrow \mathbf{b}]$ isomerization (Figure 1) [5,6].

With $\mathrm{SCF}_{3}$ reagent $\mathbf{5 a} / \mathbf{5} \mathbf{b}$, structure determination was notably challenging and solely provides a solid-state structural perspective. Thus, we wondered whether a correct structural assignment of reagent $\mathbf{5 a} / \mathbf{b}$ would have been feasible without having to resort to the preparation of crystalline congeners and/or the preparation of $\mathbf{5 b} @ \mathrm{MOF}$. Importantly, establishing a reliable way to differentiate cyclic (a) from acyclic (b) isomers in solution would facilitate future structure determination of similar iodine-based group-transfer reagents and provide greater mechanistic insight into reactivity of these reagents (Figure 1). Accordingly, we describe herein how ${ }^{17} \mathrm{O}$ NMR spectroscopy in tandem with gauge-independent atomic orbital (GIAO) calculations may be a viable approach to establishing the predominant tautomer in solution.

\section{Results and Discussion}

Arguably, the most common methods for structural elucidation of small organic molecules are one-dimensional ${ }^{1} \mathrm{H}$ and ${ }^{13} \mathrm{C}$ NMR spectroscopic techniques in combination with suitable two-dimensional experiments (COSY, HMBC, NOESY, etc.) [7]. However, in many cases (e.g., 5a versus $\mathbf{5 b}$ ) these may only provide limited information, as neither nucleus is a primary constituent of the central iodine(III) (a, X-I-O) or iodine(I) (b, O-X) motif of interest. In stark contrast, changes in the oxygen ligand's environment should be readily traceable upon oxidation from alcohol 1 to chloroiodane 2a (Figure 1, maroon), as well as during ensuing ligand substitutions, for example to fluoroiodane 3a. In particular, whether oxygen is covalently bound to iodine or another element may heavily influence its shielding and thereby provide structural information by means of ${ }^{17} \mathrm{O}$ NMR spectroscopy.

While natural abundance ${ }^{17} \mathrm{O}$ NMR has been employed previously, including the analysis of hypervalent iodine compounds [8-10], this spectroscopic method has not yet found its entry into the organic chemist's standard NMR toolbox. This, in large part, may be attributed to the extremely low natural abundance of the ${ }^{17} \mathrm{O}$ isotope $(<0.04 \%)[11,12]$. Consequently, the experiment requires high sample concentrations and relatively long

A. iodine(III)-iodine(I) tautomerism
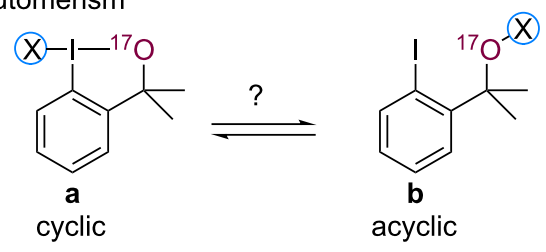

B. key structures

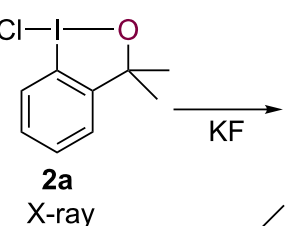

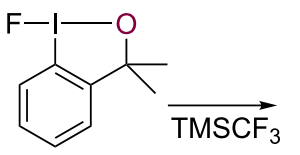

3a X-ray

$\mathrm{AgSCF}_{3}$<smiles>CC1(C)OI(C(F)(F)F)c2ccccc21</smiles>

$4 a$

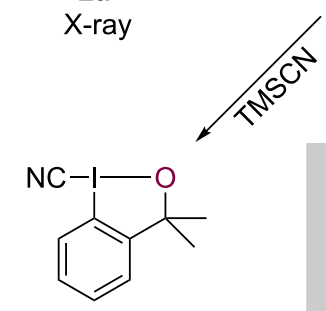

$6 a$

proposed (1995)

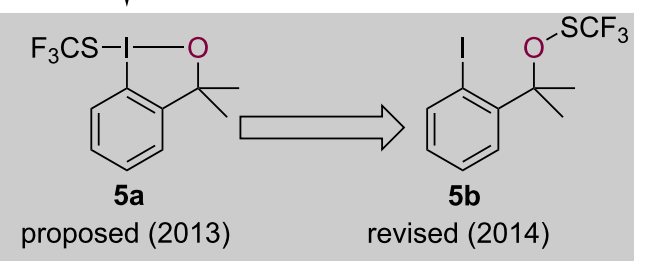

Figure 1: Tautomerism in iodine-based group-transfer reagents probed by ${ }^{17} \mathrm{O}$ NMR spectroscopy $(\mathrm{A})$ and key structures investigated herein (B) 
experimental times, and ultimately fairly broad signals are observed. Yet, due to the large chemical shift range available ( $>1000 \mathrm{ppm}$ ), the technique may still prove diagnostic, especially when paired with calculated oxygen isotropic shift values. In order to substantiate this working hypothesis, five pairs of cyclic (a) vs acyclic (b) structural isomers 2-6 were investigated initially by DFT at the $\omega$ B97XD/aug-cc-pVDZ (aug-cc-pVDZ-PP basis set for iodine [6,13]) level of theory using Gaussian 09 [14,15]. The $\omega$ B97XD functional was chosen as a reasonably cost-effective way to include long-range dispersion [14].

Geometry optimizations of both cyclic and acyclic isomers were followed by calculation of oxygen isotropic shift values $\left(\delta_{\text {iso }}\right)$ using the GIAO method (Table 1) [8-10]. Furthermore, these computed isotropic shift values $\left(\delta_{\text {iso }}\right)$ were not referenced, for example to water, since they were directly correlated to experimentally determined ${ }^{17} \mathrm{O}$ NMR shifts (vide infra). In addition, note that the calculations did not include treatment of spinorbit-induced heavy-atom effects [16]. While undoubtedly important in the framework of classical bonding paradigms, they will only have a negligible effect on oxygen shifts derived for hypervalent iodine species. Specifically, spin-orbit effects heavily depend on and propagate through s-character rich bonds. However, within classical bonding theory the hypervalent bond about iodine comprises purely of p-orbitals (RundlePimentel model) and most recently, this notion was corroborated for structure $\mathbf{4 a}$ in a computational study [17]. Hence, effects on oxygen isotropic shifts will be minor at best and systematic and therefore, be accounted for by the abovementioned referencing to experimentally determined values.

We found that the calculated $\delta_{\text {iso-values for the two isomers }}$ $\mathbf{2 a} / \mathbf{b}$ and $\mathbf{4 a} / \mathbf{b}$ differ by $\Delta \delta_{\text {iso }} \approx 20$; these differences are significantly larger for $\mathbf{3 a} / \mathbf{b}\left(\Delta \delta_{\text {iso }}=-399.1\right), \mathbf{5 a} / \mathbf{b}\left(\Delta \delta_{\text {iso }}=81.2\right)$ and $\mathbf{6 a} / \mathbf{b}\left(\Delta \delta_{\text {iso }}=52.7\right)$. Given that the larger the difference $\Delta \delta_{\text {iso }}$, the more likely a successful structural assignment based on ${ }^{17} \mathrm{O}$ NMR spectroscopy becomes, this technique may indeed prove useful for the identification of the isomeric pairs 2-6. Accordingly, spectral data on 1, 2a, 3a, 4a, 5 (assuming no assignment ), and $\mathbf{6}$ (unassigned) were acquired and further supplemented with values from some additional, structurally wellcharacterized hypervalent iodine compounds available in the literature (see Table 1 and Supporting Information File 1). Thus, a data set with a total of 11 entries was obtained.

To obtain experimental ${ }^{17} \mathrm{O}$ NMR shifts, we used samples prepared in chloroform- $d$ at a concentration of approximately 1.3 M. The obtained resonances typically featured a full-width at half maximum of around $1000-1500 \mathrm{~Hz}$ (Figure 2A). Therefore, the uncertainties of the determined ${ }^{17} \mathrm{O}$ chemical shift values $\delta_{\text {obs }}$ are rated at a minimum of $\pm 10 \mathrm{ppm}$, and thus, a reliable structural assignment should become feasible if predicted shift differences between the constitutional isomers $\mathbf{a}$ and $\mathbf{b}$ are greater. The observed ${ }^{17} \mathrm{O}$ NMR chemical shifts ranged from $32 \mathrm{ppm}$ (5) to $137 \mathrm{ppm}$ (a $\mathrm{C}_{2} \mathrm{~F}_{5}$-transfer reagent). Compounds 2a and 4a resonate at similar frequencies, with respective chemical shifts of $116 \mathrm{ppm}$ and $130 \mathrm{ppm}$. For compound 5, an

Table 1: Compilation of $\delta_{\text {iso, }} \delta_{\text {obs }}$ and $\delta_{\text {calc }}$ values.

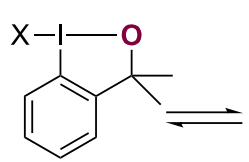

a

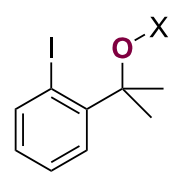

b

\begin{tabular}{|c|c|c|c|c|c|}
\hline entry & tautomer & $\delta_{\text {iso }}^{a}$ & $\delta_{o b s}{ }^{b}[p p m]$ & $\delta_{\text {calc }}^{c}[p p m]$ & $\left|\delta_{\text {calc }}-\delta_{\text {obs }}\right|$ \\
\hline 1 & - & 249.7 & 67 & 42 & 25 \\
\hline \multirow[t]{2}{*}{$2(X=C l)$} & $\mathbf{a}$ & 192.7 & 116 & 116 & 0 \\
\hline & b & 203.6 & - & 102 & - \\
\hline \multirow[t]{2}{*}{$3(X=F)$} & $\mathbf{a}$ & 236.2 & 59 & 60 & 1 \\
\hline & b & -162.9 & - & 575 & - \\
\hline \multirow[t]{2}{*}{$4\left(X=C F_{3}\right)$} & $\mathbf{a}$ & 180.1 & 130 & 132 & 2 \\
\hline & b & 203.8 & - & 101 & - \\
\hline \multirow[t]{2}{*}{$5\left(\mathrm{X}=\mathrm{SCF}_{3}\right)$} & $\mathbf{a}$ & 173.6 & - & 140 & - \\
\hline & b & 254.8 & 32 & 36 & 4 \\
\hline \multirow[t]{2}{*}{$6(X=C N)$} & $\mathbf{a}$ & 186.3 & 115 & 124 & 9 \\
\hline & b & 239.0 & - & 56 & - \\
\hline
\end{tabular}

${ }^{a} \delta_{\text {iso: }}$ computed isotropic shift value; ${ }^{b} \delta_{o b s}:$ observed (experimental) chemical shift; ${ }^{c} \delta_{\text {calc: }}$ calculated (predicted) chemical shift. 
approximately $100 \mathrm{ppm}$ smaller chemical shift value was observed with $\delta_{\text {obs }}=32 \mathrm{ppm}$, and for unassigned structure 6 we measured $115 \mathrm{ppm}$. It is noteworthy to indicate that under certain circumstances the absolute ${ }^{17} \mathrm{O}$ NMR shift alone may be misleading in structure determination. For instance, the experimental value of $59 \mathrm{ppm}$ for the known cyclic fluoroiodinane $\mathbf{3 a}$ is closer to the observed values of acyclic $\mathbf{1}$ (67 ppm) and $\mathbf{5 b}$ (32 ppm) than it is to cyclic 2a and 4a. However, assessment of the DFT-calculated isotropic shift values $\left(\delta_{\text {iso }}\right)$ in tandem with experimental ${ }^{17} \mathrm{O}$ NMR data $\left(\delta_{\text {obs }}\right)$ lends credence to the aforementioned structural assignment. Specifically, for the "unassigned" compounds mentioned above, the best $\mathrm{R}^{2}$-value for a linear relationship $\delta_{\text {obs }} \sim \delta_{\text {iso }}$ is obtained when $\mathbf{5}$ and $\mathbf{6}$ are assigned as $\mathbf{5 b}$ and $\mathbf{6 a}$, where the additional known compounds serve as calibration (Figure 2B) [18]. Based on the thus derived equation, ${ }^{17} \mathrm{O}$ NMR chemical shifts $\delta_{\text {calc }}$ can be predicted for both isomers. A notable exception is the free alcohol $\mathbf{1}$, which is not part of the linear relationship and consequently displays a large residual value (Table 1). Conceivably, this may be due to intermolecular hydrogen bonding with the solvent or other alcohol molecules in the concentrated solution. In fact, including a methanol solvent molecule as a hydrogen-bond donor in the DFT calculation will shift the $\delta_{\text {calc }}$ in the right direction for 1 (i.e., to $\delta_{\text {calc }}=47 \mathrm{ppm}$ based on $\delta_{\text {iso }}=245.8$, although $\left|\delta_{\text {calc }}-\delta_{\text {obs }}\right|$ is still $\left.20 \mathrm{ppm}\right)$.

For the pair $\mathbf{5 a} / \mathbf{b}$ a difference $\Delta \delta_{\text {calc }}$ of $104 \mathrm{ppm}$ is obtained and a value of $68 \mathrm{ppm}$ results for $\mathbf{6 a} / \mathbf{b}$ (Figure 2). Both figures are significantly larger than the ${ }^{17} \mathrm{O}$ chemical shift's lower-bound uncertainty estimate of $\pm 10 \mathrm{ppm}$. While $\mathbf{5 a} / \mathbf{b}$ indeed has been shown to exist as the thioperoxide $\mathbf{5 b}$ (vide infra), a crystallographic study on $6 \mathbf{a} / \mathbf{b}$ is required to corroborate our prediction as $6 \mathbf{a}$.

To further gauge the utility of this approach, the activation of Togni reagent 4a was studied, in particular its protonation with a strong acid [1]. This brings about a significant elongation of the I-O bond from 2.203(5) $\AA$ in $4 \mathbf{a}$ to 2.4991(13) $\AA$ in the fully protonated form $\mathbf{4 c}[1,19]$. Most recently, Toste and co-workers studied this activation strategy too and demonstrated that in the presence of an equivalent of gaseous $\mathrm{HCl}$ compound $\mathbf{4 a}$ afforded an isolable iodonium-type structure [20]. Although this activation can be conveniently followed by ${ }^{19} \mathrm{~F}$ NMR spectroscopy with 4 a resonating at $-40.1 \mathrm{ppm}$ and the fully protonated "iodonium" congener $4 \mathbf{c}$ at $-20 \mathrm{ppm}$ [1], this technique provides no indication on how to best represent $4 \mathbf{c}$ in solution. Does the compound resemble the molecular structure obtained in the solid state with oxygen still coordinated to iodine or would a free alcohol be a more accurate representation? In order to generate $\mathbf{4 c}$, reagent $4 \mathbf{a}$ was treated with five equivalents of trifluoroacetic acid (TFA) and then subjected to spectroscopic

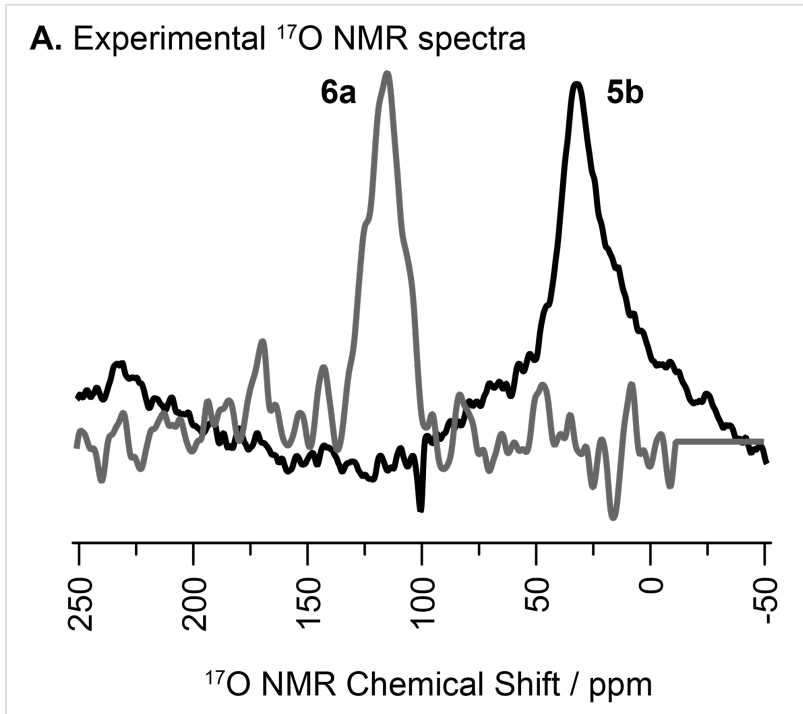

B. Linear regression analysis $\delta_{o b s} \sim \delta_{\text {iso }}$

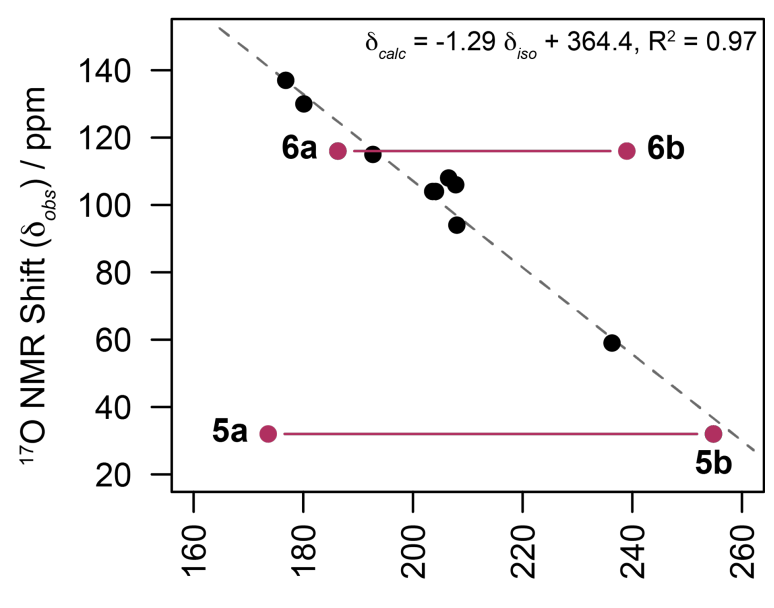

Isotropic Shift Value $\left(\delta_{\text {iso }}\right)$

Figure 2: Assignment of acyclic (b) and cyclic (a) structures to 5 and 6 , respectively, based on computed isotropic shift values $\left(\delta_{\text {iso }}\right)$ and experimental ${ }^{17} \mathrm{O}$ NMR chemical shifts $\left(\delta_{\text {obs }}\right)$.

analysis. A ${ }^{19} \mathrm{~F}$ NMR chemical shift of -23.2 ppm was obtained, thereby confirming the presence of $4 \mathbf{c}$. However, under these strongly acidic and activating conditions, the compound is unstable over a prolonged period of time (12 h). During the acquisition of the ${ }^{17} \mathrm{O}$ NMR data, approximately $36 \%$ of $4 \mathbf{c}$ had decomposed to the corresponding $\alpha$-methylstyrene derivative as indicated by ${ }^{1} \mathrm{H}$ NMR spectroscopy (see Supporting Information File 1). As this byproduct is ${ }^{17} \mathrm{O}$ NMR silent, the spectral data acquisition was unhampered and a chemical shift $\delta_{\text {obs }}=77 \mathrm{ppm}$ was measured. This value is larger than the chemical shift obtained for the free alcohol 1 (67 ppm) and at the same time, also significantly smaller than the value obtained for the native reagent $4 \mathbf{a}(130 \mathrm{ppm})$. Structure $4 \mathbf{c}$ was computed in the gas phase in absence of a counter anion and ge- 
ometry optimization furnished a minimum reminiscent of the pictographic representation of $\mathbf{4} \mathbf{c}$ with an intact but significantly elongated I-O bond of $2.55 \AA$ (Figure 3 ) and qualitatively, the NMR data are in support of this notion. From a quantitative point of view, the data points $\left(\delta_{\text {iso }}, \delta_{\text {obs }}\right)$ for $\mathbf{1}, \mathbf{4 b}$, and $\mathbf{4 c}$ afforded a perfect linear correlation with $\mathrm{R}^{2}=1$, thus lending further credence to the representation of the protonated form $\mathbf{4 a}$ in solution as $\mathbf{4 c}$ (see Supporting Information File 1).

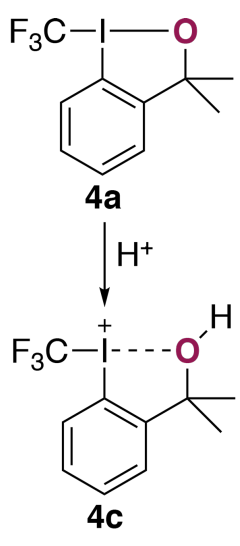

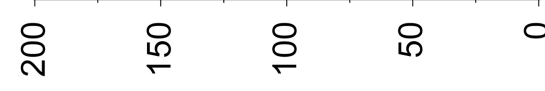 \\ ${ }^{17} \mathrm{O}$ NMR Chemical Shift / ppm}

Figure 3: Protonation of 4 a with trifluoroacetic acid (5 equiv) affords 4c, followed by ${ }^{17} \mathrm{O}$ NMR spectroscopy.

\section{Conclusion}

In summary, the present study demonstrates that ${ }^{17} \mathrm{O}$ NMR spectroscopy at natural abundance coupled with DFT-calculated isotropic shift values can be used to gain insight into the solution-phase tautomerism observed in iodine-based grouptransfer reagents. In particular, we confirm that Shen's revised, electrophilic $\mathrm{SCF}_{3}$-transfer reagent adopts an "acyclic" thioperoxide tautomeric form in solution whereas an electrophilic cyanide source prefers the "cyclic" iodane. Since ${ }^{17} \mathrm{O}$ NMR experiments are easily implemented on contemporary spectrometers, this method may provide the most convenient spectroscopic handle to re-evaluate known structures, facilitate further mechanistic studies, and provide a complimentary approach to solid-state structural analysis.

\section{Supporting Information}

\section{Supporting Information File 1}

${ }^{17} \mathrm{O}$ NMR spectra and calculated molecular geometries. [https://www.beilstein-journals.org/bjoc/content/ supplementary/1860-5397-14-203-S1.pdf]

\section{Acknowledgements}

This work was generously supported by the Swiss National Science Foundation (N. S., P3P3P2_167744), ETH Zürich Postdoctoral Fellowship Program (C. R. P.) and the National Science and Engineering Research Council of Canada (B. J. J.). The authors gratefully acknowledge the support of Prof. Dr. D. Günther and Prof. Dr. A. Togni (ETH Zürich).

\section{ORCID ${ }^{\circledR}$ iDs}

Nico Santschi - https://orcid.org/0000-0003-3839-778X Cody Ross Pitts - https://orcid.org/0000-0003-1047-8924 Benson J. Jelier - https://orcid.org/0000-0002-5531-0431 René Verel - https://orcid.org/0000-0003-1575-2232

\section{References}

1. Charpentier, J.; Früh, N.; Togni, A. Chem. Rev. 2015, 115, 650-682. doi:10.1021/cr500223h

2. Shao, X.; Wang, X.; Yang, T.; Lu, L.; Shen, Q. Angew. Chem., Int. Ed. 2013, 52, 3457-3460. doi:10.1002/anie.201209817

3. Shao, X.; Xu, C.; Lu, L.; Shen, Q. Acc. Chem. Res. 2015, 48, 1227-1236. doi:10.1021/acs.accounts.5b00047

4. Vinogradova, E. V.; Müller, P.; Buchwald, S. L. Angew. Chem., Int. Ed. 2014, 53, 3125-3128. doi:10.1002/anie.201310897

5. Sun, T.-Y.; Wang, X.; Geng, H.; Xie, Y.; Wu, Y.-D.; Zhang, X.; Schaefer, H. F., III. Chem. Commun. 2016, 52, 5371-5374. doi:10.1039/C6CC00384B

6. Koichi, S.; Leuthold, B.; Luthi, H. P. Phys. Chem. Chem. Phys. 2017, 19, 32179-32183. doi:10.1039/C7CP05943D

7. Pretsch, E.; Bühlmann, P.; Badertscher,, M. Structure Determinations of Organic Compounds; Springer Verlag GmbH: Berlin, Germany, 2015.

8. Mocci, F.; Uccheddu, G.; Frongia, A.; Cerioni, G. J. Org. Chem. 2007, 72, 4163-4168. doi:10.1021/jo070111h

9. Fusaro, L.; Luhmer, M.; Cerioni, G.; Mocci, F. J. Org. Chem. 2009, 74, 8818-8821. doi:10.1021/jo901841v

10. Fusaro, L.; Mocci, F.; Luhmer, M.; Cerioni, G. Molecules 2012, 17, 12718-12733. doi:10.3390/molecules171112718

11. Boykin, D. W. 170 NMR Spectroscopy in Organic Chemistry; CRC Press: Boca Raton, Florida, USA, 1991.

12. Berger, S.; Braun, S. 200 and More NMR Experiments: A Practical Course; Wiley-VCH: Weinheim, Germany, 2004.

13. Peterson, K. A.; Figgen, D.; Goll, E.; Stoll, H.; Dolg, M. J. Chem. Phys. 2003, 119, 11113-11123. doi:10.1063/1.1622924

14. Chai, J.-D.; Head-Gordon, M. Phys. Chem. Chem. Phys. 2008, 10, 6615-6620. doi:10.1039/b810189b

15. Gaussian 09, Revision D.01; Gaussian, Inc.: Wallingford, CT, 2013.

16. Kaupp, M.; Malkina, O. L.; Malkin, V. G.; Pyykkö, P. Chem. - Eur. J. 1998, 4, 118-126. doi:10.1002/(SICI)1521-3765(199801)4:1<118::AID-CHEM118>3.0.CO ;2-6

17. Pinto de Magalhães, H.; Lüthi, H. P.; Bultinck, P. Phys. Chem. Chem. Phys. 2016, 18, 846-856. doi:10.1039/C5CP05343A

18. Note that calibrations using other functionals may provide inferior coefficients of determination. For instance, using the same basis set at B3LYP, $R 2 \approx 0.49$. 
19. Liebing,, P.; Pietrasiak, E.; Otth, E.; Kalim, J.; Bornemann, D.; Togni, A. Eur. J. Org. Chem. 2018, 3771-3781. doi:10.1002/ejoc.201800358

20. Brantley, J. N.; Samant, A. V.; Toste, F. D. ACS Cent. Sci. 2016, 2 , 341-350. doi:10.1021/acscentsci.6b00119

\section{License and Terms}

This is an Open Access article under the terms of the Creative Commons Attribution License

(http://creativecommons.org/licenses/by/4.0). Please note that the reuse, redistribution and reproduction in particular requires that the authors and source are credited.

The license is subject to the Beilstein Journal of Organic Chemistry terms and conditions:

(https://www.beilstein-journals.org/bjoc)

The definitive version of this article is the electronic one which can be found at:

doi:10.3762/bjoc. 14.203 